\title{
Heat Transfer in an Axially Rotating Tube Fitted with Twin Twisted Tapes*
}

\author{
Shyy Woei CHANG**, Yih Jena $\mathrm{JAN}^{* * *}$ and Lo May SU***
}

\begin{abstract}
This paper describes an experimental study of heat transfer in an axially rotating tube fitted with twin twisted tapes. The manner in which rotation modifies the forced heat convection is considered for the case where the tube rotates about an axis in parallel to the tube's axis of symmetry with particular reference to the design of enhanced cooling channels for rotor windings in a rotating electro-machinery. A selection of experimental results illustrates the individual and interactive effects of Coriolis and centripetal buoyancy forces on heat transfer along the radially outer edge of rotating tube. With the prevailing swirl-flow structures generated by twin twisted tapes, the isolated Coriolis force effect plays a dominant role to initiate the heat transfer reduction form the static-tube scenario that is followed by a subsequent recovery which could lead to heat transfer improvement as the relative strength of Coriolis force increases. The reversed buoyancy impact from improving to impeding heat transfer develops at the higher level of Coriolis force. An empirical correlation, which is physically consistent, has been developed to permit the evaluation of interactive effects of swirling-flows, convective inertial force, Coriolis force and centripetal buoyancy on heat transfer.
\end{abstract}

Key Words: Axially Rotating Flow, Twin Twisted Tapes, Rotor Winding Cooling

\section{Nomenclature}

English symbols

$B u:$ Buoyancy number $=\beta_{0} \tau d$

Co $:$ Coriolis number $=N d / W_{m}$

$C_{p}:$ Specific heat of fluid $\left[\mathrm{Jkg}^{-1} \mathrm{~K}^{-1}\right]$

$d:$ Hydraulic diameter of test duct $[\mathrm{m}]$

$f_{B}, f_{C}$ : Functions of Coriolis number

$H:$ Eccentricity [m]

$k$ : Thermal conductivity of fluid $\left[\mathrm{Wm}^{-1} \mathrm{~K}^{-1}\right]$

$N$ : Rotating speed of duct $\left[\mathrm{rads}^{-1}\right]$

$\mathrm{Nu}$ : Rotational Nusselt number

$N u_{0}$ : Stationary Nusselt number

$N u_{\infty}$ : Nusselt number value for stationary developed turbulent duct flow

* Received 20th October, 2003 (No. 03-5129)

** Thermal Fluids Laboratory, National Kaohsiung Marine University, No.142, Hai-Chuan Road, Nan-Tzu District, Kaohsiung, Post code: 811, Taiwan, R.O.C. E-mail: swchang@mail.nkmu.edu.tw

*** Department of Marine Engineering, National Kaohsiung Marine University, No.142, Hai-Chuan Road, Nan-Tzu District, Kaohsiung, Post code: 811, Taiwan, R.O.C.

**** Department of Electrical Engineering, Tung Fang College of Technology and Commerce, No.110, Tung Fang Road, Hu-Nei Shang, Kaohsiung Hsien, Taiwan, R.O.C. $n:$ Exponent

$P$ : Dimensionless pressure of flow in rotating duct

$p$ : Pressure of flow in stationary duct $\left[\mathrm{Nm}^{-2}\right]$

$p^{*}$ : Pressure of flow in rotating duct $\left[\mathrm{Nm}^{-2}\right]$

Pr : Prandtl number $=\mu C_{p} / k$

$q$ : Convective heat flux $\left[\mathrm{Wm}^{-2}\right]$

$R e:$ Reynolds number $=\rho W_{m} d / \mu$

$R i:$ Richardson number $=g \beta\left(T_{w}-T_{f}\right) d / W_{m}^{2}$

$\vec{r}$ : Position vector $[\mathrm{m}]=x \vec{i}+y \vec{j}+z \vec{k}$

$\vec{R}$ : Dimensionless position vector $=X \vec{i}+Y \vec{j}+Z \vec{k}$

$T$ : Fluid temperature $[\mathrm{K}]$

$T_{0}$ : Fluid reference temperature [K]

$T_{f}$ : Fluid bulk temperature $[\mathrm{K}]$

$T_{w}$ : Wall temperature of test duct [K]

$\vec{V}$ : Dimensionless flow velocity vector

$\vec{v}$ : Flow velocity $\left[\mathrm{ms}^{-1}\right]$

$W_{m}$ : Mean through flow velocity $\left[\mathrm{ms}^{-1}\right]$

$x, y, z$ : Coordinates of rotating reference frame

$x:$ Axial location $[\mathrm{m}]$

$X, Y, Z$ : Dimensionless coordinates of rotating reference frame $(x / d, y / d, z / d)$

Greek symbols

$\beta$ : Thermal expansion coefficient of fluid $\left[\mathrm{K}^{-1}\right]$

$\beta_{0}$ : Thermal expansion coefficient of fluid at reference temperature $\left[\mathrm{K}^{-1}\right]$

$\vec{\omega}$ : Angular velocity of rotating duct $\left[\mathrm{s}^{-1}\right]$ 
$\vec{\Omega}$ : Dimensionless angular velocity vector of rotating duct

$\rho:$ Fluid density $\left[\mathrm{kgm}^{-3}\right]$

$\rho_{0}$ : Fluid density at reference temperature $\left[\mathrm{kgm}^{-3}\right]$

$\mu$ : Fluid dynamic viscosity $\left[\mathrm{kgs}^{-1} \mathrm{~m}^{-1}\right]$

$\varepsilon$ : Dimensionless eccentricity

$\tau$ : Streamwise gradient of flow bulk temperature $\left[\mathrm{Km}^{-1}\right]$

$\eta$ : Dimensionless fluid temperature

$\Psi, \Phi, \phi:$ Unknown functions

\section{Introduction}

The inefficiency of stator and rotor windings in a rotating electro-machinery with high power density converts considerable amount of electrical energy into thermal losses, which necessitates the reliable cooling channels inside these windings in order to ensure the reliability of electrical insulation. A variety of assorted coolant circuit configurations for the rotor windings involves a combination of axial passages, inter-connections and plenum chambers which are rotated about the radial axis. In addition to the channel-geometry induced flow complexity, the Coriolis and centrifugal forces are generated in the axially rotating channel that further modifies the heat transfer phenomena from the static scenarios. The numerical and experimental studies for the axially rotating ducts of circular, square and rectangular sectioned channels were reported by Morris and his group ${ }^{(1)-(7)}$. The rotation generates a conservative field of centrifuge directing toward the outer edge of an axially rotating channel. For isothermal flow, no secondary flow generated by the centrifugal forces but a cross stream forced vortex-type radial equilibrium pressure field is accordingly established ${ }^{(1)}$. Because the rotating vector is in the axial direction, the axial flow velocity has no contribution of creating Coriolis acceleration and the vorticity generation as the Coriolis force also vanishes identically in fully developed flow regime. When the densities of fluids vary in the axial, angular and radial directions due to heat transfer, the centripetal buoyancy forces arise to drive the tube-core cold coolants toward the outer edge of rotating tube with a pair of heated, less dense, circumferential return flows washes along the tube wall from outer edge toward inner edge. A variety of cross-stream secondary flows ${ }^{(3),(8)}$ and axial circulations ${ }^{(4)}$ are initiated by the centripetal buoyancy forces so that the flow velocities in the radial and angular directions are accordingly established that also incurs the Corioliscentrifuge interactions which are capable of distorting the secondary flow profile generated by the centripetal buoyancy forces ${ }^{(4)}$. These studies ${ }^{(1)-(8)}$ have established that rotation could considerably improve heat transfers from the static references due to the influence of the crossstream secondary flows arising out of rotation. The asymmetric temperature profile produced as a consequence of rotation results in a corresponding circumferential variation in local heat transfer along the circumference of tube wall $^{(1)}$. When the Coriolis-centrifuge interactions prevail over a heated axially rotating channel, the centripetal buoyancy effect could be reversed from improving to impeding heat transfer ${ }^{(4)}$. In the quest of seeking for heat transfer augmentation in a rotating tube, the elliptical geometry has been shown to be superior to others that are frequently used as coolant channels in rotating machinery ${ }^{(9)}$. Nevertheless, the twisted tape inserts have been widely used in static ducted-flow as a passive heat transfer augmentation measure. Heat transfer augmentation in such a swirl tube mainly utilizes the secondary fluid circulation that is induced by the centrifugal force when the flow travels in a twisted passage. Depending on the flow rate and twisted tape geometry, the heat transfer enhancement can be additionally attributed from the blockage of flow, the fin effect of insert and the elongated twisted flow path ${ }^{(10)-(15)}$. Although the use of twisted tape inserts has been the subject of much fundamental research over the years for static duct flow situations, no information is available in the open literature when the twin twisted plates are inserted in a static or rotating tube. As the twisted tape inserts generate the secondary flows ${ }^{(10),(15)}$ which necessitate the Coriolis accelerations even in the developed flow regime, the complex Coriolis-centrifuge interactions are expected in an axially rotating tube fitted with twisted tape insert(s). Such Coriolis-centrifuge interactions coupled with buoyancy effects in an axially rotating swirl tube could dramatically modify the flow structure and hence the heat transfer established in the static condition. Under these circumstances the improved understanding of these phenomena could assist for confident design measures.

The present paper describes the results of a series of experiments aimed at studying the effect of axial rotation on heat transfer in a circular tube fitted with twin continuously twisted tapes. When the channel remains static, the pair of twisted tapes creates streamwise swirls that improve heat transfer from the smooth-walled duct flow. When the swirl tube rotates axially, the twisted tape-induced centrifuge interacts with Coriolis force and centripetal buoyancy that further modifies the thermal fluids phenomena. It is with the combined effect of the twin twisted tapes and the rotation that the present investigation is concerned.

\section{Experimental Strategy}

In addition to the geometric and thermal/flow boundary conditions simulated by the heat transfer test section, the dimensionless parameters governing the thermal fluids phenomena in an axially rotating tube is identified from the non-dimensional flow momentum and energy equations. The adoption of Navier-Stoke concept for deriving the vector form of the flow momentum equation in an ax- 
ially rotating heated duct referred to a synchronously rotating frame of reference indicated in Fig. 2 gives $^{(1)}$ :

$$
\begin{aligned}
& \frac{D \vec{v}}{D t}=-\frac{1}{\rho} \nabla p^{*}+\mu \nabla^{2} v-2 \vec{\omega} \times \vec{v} \\
& \quad-\beta\left(T-T_{0}\right) \vec{\omega} \times(\vec{\omega} \times[\vec{r}+H \vec{j}])
\end{aligned}
$$

All symbols are defined in the nomenclature. The last two terms of Eq. (1) arise from the Coriolis and centripetal accelerations respectively. The dynamic natures of Coriolis and centripetal buoyancy force-components are demonstrated by their own vector identities in Eq. (1). As the tube rotates axially, the rotating vector, $\vec{\omega}$, in Eq. (1) is in the axial direction. Therefore, the axial flow velocity has no contribution of generating Coriolis acceleration. Nevertheless, for the present axially rotating tube within which twin twisted plates are inserted, the two pairs of spiral flow paths constructed by the tube wall and twisted plates ensure that the flow-velocity components in the radial and angular directions prevail over the complete length of test tube. Thus the Coriolis accelerations are generated by the radial and angular flow velocity components in terms of $2 \vec{\omega} \times \vec{v}$ over the entire flow domain, which could consequently modify the flow and heat transfer inside the present rotating test tube. Equation (1) retains Boussinesq approximation for the centripetal acceleration only so that the buoyancy interaction is limited with the centrifuge. The so-called centripetal acceleration, $\vec{\omega} \times(\vec{\omega} \times[\vec{r}+H \vec{j}])$, is hydrostatic in nature that always orients radially outward. When this acceleration component couples with the fluid temperature field, $T(x, y, z)$, a natural-buoyancy type of flow described as $\beta\left(T-T_{0}\right) \vec{\omega} \times(\vec{\omega} \times[\vec{r}+H \vec{j}])$ is induced that creates a pair of cross-plane secondary flows. Because the temperature of coolant, which is controlled by the flow energy equation, varies over the flow field, the effect of centripetal acceleration no longer remains hydrostatic in a rotating heated channel. Substitution of the non-dimensional parameters defined in Eq. (2) into the momentum and energy equations of flow with the coordinates referred to the axially rotating frame of reference, $x y z$, obtains the dimensionless equations (3) and (4) which, respectively, express the conservation laws of momentum and energy for the rotating flow.

$$
\begin{aligned}
& \left\{\begin{array}{l}
\vec{V}=\frac{\vec{v}}{W_{m}}, \quad \vec{\Omega}=\frac{\vec{\omega}}{N}, \quad \eta=\frac{T-T_{0}}{\tau d}, \\
P=\frac{p^{*}}{\rho W_{m}^{2}}, \quad \varepsilon=\frac{H}{d}, \quad \vec{R}=X \vec{i}+Y \vec{j}+Z \vec{k}
\end{array}\right. \\
& \frac{D \vec{V}}{D t}=-\nabla P^{*}+\frac{1}{\operatorname{Re}} \nabla^{2} \vec{V}-C o(2 \vec{\Omega} \times \vec{V}) \\
& -C o^{2} B u \eta(\vec{\Omega} \times[\vec{\Omega} \times(\vec{R}+\varepsilon \vec{j})]) \\
& \frac{D \eta}{D t}=\frac{1}{\operatorname{RePr}} \nabla^{2} \eta
\end{aligned}
$$

All symbols quoted in Eqs. (2) - (4) are defined in the nomenclature section. The $\tau$ value is the streamwise flowbulk-temperature gradient which varies with the heating level at a specified coolant mass flow rate that reflects the strength of buoyancy. The governing flow parameters appeared in the dimensionless flow momentum and energy equations are collected in Eqs. (5) - (8).

$$
\begin{aligned}
\operatorname{Re}=\frac{\rho W_{m} d}{\mu} & \text { Reynolds number } \\
\operatorname{Co}=\frac{N d}{W_{m}} & \text { Coriolis number } \\
B u=\beta_{0} \tau d & \text { buoyancy number } \\
\operatorname{Pr}=\frac{\mu C_{p}}{k} & \text { Prandtl number }
\end{aligned}
$$

In the static duct, the forced convective mechanism is characterized by the usual pipe flow Reynolds number, $R e$, that quantifies the inertial-to-viscous forces ratio. In the axially rotating duct, the Coriolis number, $\mathrm{Co}$, is derived to quote the relative strength of Coriolis force to inertial force. The buoyancy number, $B u$, which always couples with $\mathrm{Co}^{2}$, has its origin in the interaction between the temperature dependent fluid density and centripetal forces that characterizes the strength of centripetal buoyancy in a manner analogous to the free convection drive by the earth gravity. To close Eqs. (2) and (3) for flow and temperature solutions in an axially rotating pipe requires a set of geometrical parameters such as the number and description of twisted plates, the flow and thermal boundary conditions. These boundary conditions are pre-defined once the experimental apparatus is built. The variation of any governing parameters, such as $R e, C o, B u$ and $P r$ in Eqs. (2) and (3), results in different solutions of flow and temperature fields. Accordingly the heat convective capability in the axially rotating pipe varies. Such heat transfer variation corresponding to a systematic change of each of the governing flow parameters in Eqs. (5) to (8) is experimentally recorded by the tube-wise measurements of local heat transfer values. A following parametric analysis based on these experimental results could reveal the heat transfer physics in associating with each varying parameter. Within the temperature range tested by the present study, the maximum $\operatorname{Pr}$ variations are negligible at the levels about $1.06 \%$. Therefore the strategic approach of this study is to disclose the functional relationship of

$$
N u=\Psi\{R e, C o, B u, X\}
$$

where the local Nusselt number, $\mathrm{Nu}$, is experimentally defined as

$$
N u=\frac{q}{k\left(T_{w}-T_{f}\right)}
$$

The convective heat flux, $q$, in Eq. (10) is obtained by subtracting the external heat loss from the total heat flux generated. A set of pre-calibration runs aimed at revealing the functional relationships of external heat loss with the wall-to-ambient temperature difference and the rotating speed is performed. Having defined the convective heat 
flux, $q$, the one-dimensional finite difference representation of Fourier conduction law is applied to correct the wall-temperature measurement to the inner plain-wall surface. The local enthalpy balance is also accounted at each measurement location to determine the flow bulk temperature, $T_{f}$. All the fluid properties used to define the dimensionless parameters in Eq. (9) are consequently evaluated at the local flow bulk temperature level. The description of the experimental program formulated to study the effects of rotation on heat transfer in an axially rotating tube fitted with twin twisted plates now follows.

\subsection{Rig simulation}

2.1.1 Rotating test facility Figure 1 shows the schematic details of the rotating rig used for the present investigation. The rotor assembly consists of a hollow shaft (1) connected to a rotating test platform (2), which is supported by two main bearings (3). The heated test section (4) is supported by a pair of webbing tubes (5) that respectively connect with the coolant's entry and exit ports on the rotating test platform. The right-hand-side of the shaft (as indicated in Fig. 1) has a centrally located blind bore to permit air to enter the shaft via an EKK high pressure rotary seal assembly (6). The pressurized air then flows through the webbing tube and the test section, which returns to a centrally located blind hole bored on the lefthand-side of the hollow shaft. In this channel, the test air can enter the rotor, pass through the heat transfer test section and exit from the hollow shaft at the left-hand end of rotor. Prior to entering the rotating test rig, the air is pressurized by IWATA SC $175 \mathrm{C}$ screw-type compressor unit after which the flow is dehumidified and cooled to the ambient temperature through a refrigerating system. The test coolant is then channeled into the rotor assembly through a dryer, a pressure regulator and filter, a pressure transducer, a Tokyo Kesio TF-1120 mass flow meter and a needle valve for the control of mass flow rate. The nominal through flow Reynolds number is controlled by ad-
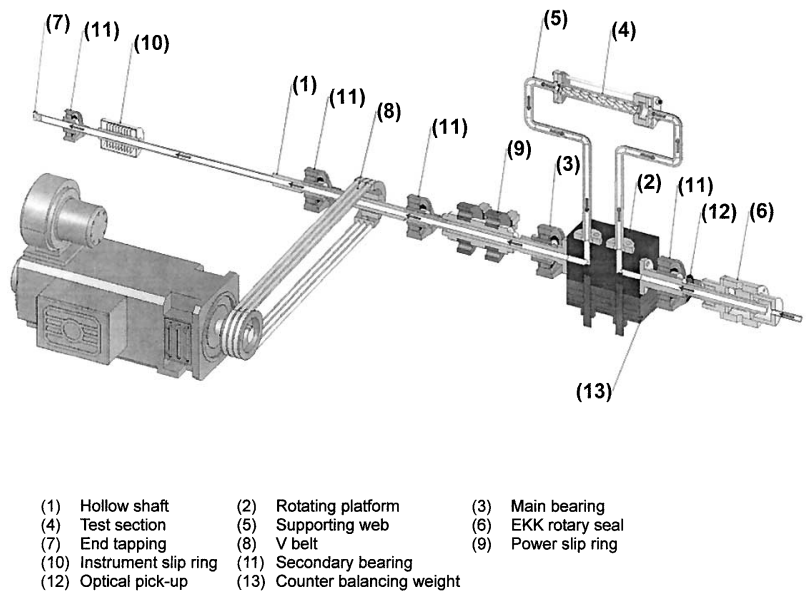

Fig. 1 Rotating test rig justing the mass flow rate measured at $30 \mathrm{~m}$ upstream of the rotating rig. Because the coolant properties, such as viscosity and density, varied with the local fluid temperature, the mass flow rate is adjusted to compensate the fluidproperty variations that controls the variations of Reynolds and Coriolis numbers at the flow entrance of test section within $\pm 1 \%$ of the nominal values. To permit the control of pressure level in the test section, a number of choked orifices, bored through the tapping threads (7), are fitted into the left-hand end of hollow shaft (1), which is interchangeable in order to match the text matrix with a predefined set of Reynolds and Coriolis numbers. The rotor assembly is driven by a speed-controlled DC electric motor using three $\mathrm{V}$ belts (8). For the present study, the pressure levels are controlled in the range of 1.23-5.21 bars in order to cover the full test matrix. Two electrical power slip rings (9) are capable of transferring electrical current up to 200 Amps from a Variac transformer to the electrical heater in the test section. A silver/silvergraphite instrumentation slip ring assembly (10) transfers the electrical signals from thermocouples attached to the heated surface to the computer for data storage and processing via the Fluke Net-Daq 2640A data logger. The section that consists of the power slip rings, the driven pulley and the instrument slip ring is supported by two secondary bearings (11). The speed of the rotor is measured using an optical pick-up and encoder unit (12) attached to the shaft. The rotor assembly is dynamically balanced by the counter weight (13) secured on the back of rotating platform.

2.1.2 Heat transfer test section Figure 2 shows the constructional details of heat transfer test section. The complete test section is mounted between two supporting

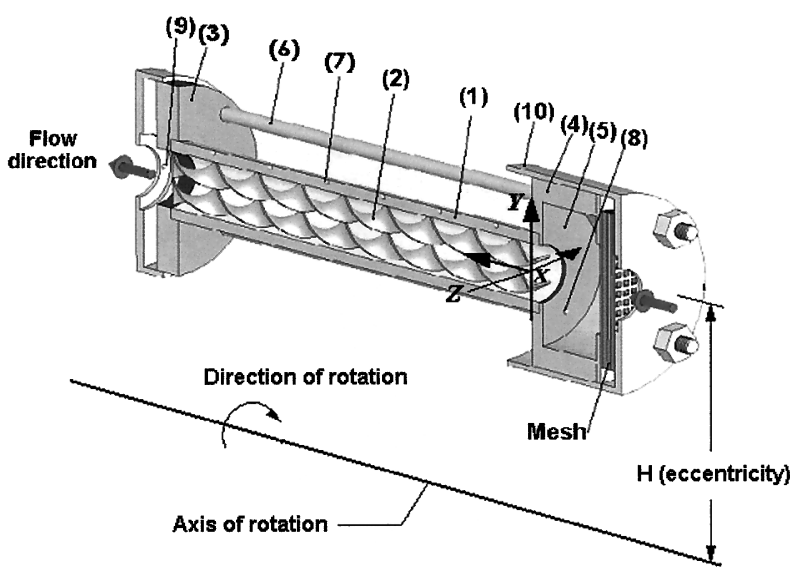
(1) Test tube
(2) Twisted tape
(5) Plenum chamber
(3) Insulating bush
(4) End bush
(7) Thermocouple
(8) Thermocouple measuring flow entry temperature
9) Thermocouple measuring flow exit temperature
(10) Stiffing tube

Fig. 2 Heat transfer test section 
webs that provide an eccentricity $(\mathrm{H})$ of $600 \mathrm{~mm}$ from the center of rotation as indicated in Fig. 2. The test tube (1) is made from seamless stainless tube that has a bore diameter of $28 \mathrm{~mm}$, a wall thickness of $2.5 \mathrm{~mm}$ and a nominal length of $290 \mathrm{~mm}$. Two continuous and longitudal twisted tapes (2) with width, pitch and thickness of 14,25 , and $1 \mathrm{~mm}$ respectively are fitted in the test tube. The rationale of using twin twisted plates instead of single twisted plate was based on our previous test results that compared the heat transfer rates between two static tubes fitted with single and twin twisted plates. This series of tests showed that the local Nusselt numbers along the static tube fitted with twin twisted tape were consistently higher than the tube fitted with single twisted tape. As shown in Fig. 2, these two twisted plates are both positioned to be aligned in the radially outward direction at the entrance of test tube. Two insulating bushes (3) and (4) hold the plenum chamber (5) and test tube by four draw bolts (6) to give a heated length of $275 \mathrm{~mm}$ between the end bush and plenum chamber. A pair of twin start threads having the same pitch but different depths is machined onto the outer surface of the test tube to facilitate the installation of thermocouples and electrical heating wire. The deeper thread is used for embedding seven equally spaced $\mathrm{K}$ type thermocouples (7) along the radial outer edge of the tube. The depth of this thread is arranged to keep each thermocouple sensing junction $0.5 \mathrm{~mm}$ away from the inner surface of test tube. Coolant leakage from the contacting surfaces between the constructional parts is prevented by means of a series of internal $\mathrm{O}$ ring seals. Two additional thermocouples (8) and (9) penetrate into the core of plenum chamber and the channel in the exit bush to measure the flow entry and exit temperatures respectively. An aluminum tube (10) is used to stiffen the final assembly. In order to minimize external heat loss during heat transfer/loss experiments, the annual space between the heated test tube and stiffening tube is filled with thermal insulation material.

\subsection{Experimental programs}

The program involves three phases. The static flow conditions in the first phase are initially examined to generate the database against which the rotating results obtained at the second phase could be compared to unravel the rotational effects on heat transfer. It is followed by a parametric analysis of the interactive and individual effects of rotating forces with and without buoyancy interaction on heat transfer. In the third phase, a methodology is proposed to devise the physically consistent heat transfer correlation. At each selected set of Reynolds and Coriolis numbers tested, five different levels of heating power, which respectively heated the wall temperature at the exit plane $(X=7.92)$ to the levels of $338 \mathrm{~K}, 358 \mathrm{~K}$, $383 \mathrm{~K}, 408 \mathrm{~K}$ and $433 \mathrm{~K}$. Each set of heat transfer data generated in this manner compares the influences of buoyancy on heat transfer at the predefined Re-Co combina- tion. The rotating speeds in the range of $0-1000 \mathrm{rev} / \mathrm{min}$ are adjusted to control the Coriolis numbers at Reynolds numbers of $5000,7500,10000,12500$ and 15000 at the precise values of $0,0.1,0.3,0.5,0.7$ and 1.0. The buoyancy levels examined in terms of buoyancy number defined in Eq. (7) are in the range of $0.000675-0.01507$. To express the buoyancy level relative to the inertial force, the range of Richardson number examined was evaluated as $0.00047-0.1057$. For each individual set of tests, the flow and heating level are fixed for about 45 minutes in order to assure the steady state condition. When the differences between the successive time-averaged wall temperature measurements remain within $\pm 0.3 \mathrm{~K}$, the steadystate is assumed and the on-line data acquisition system is activated. These raw experimental data are subsequently processed to generate the appropriate Reynolds, Coriolis, and buoyancy numbers; and the individual effect of these parameters on the Nusselt number distribution examined. The uncertainty approximation of the data reduction was conducted $^{(16)}$. The temperature measurements were the major sources to attribute uncertainty. With the temperature difference between the wall and fluid varied from 29 to $105 \mathrm{~K}$, the maximum uncertainty for the $\mathrm{Nu}, \mathrm{Re}, \mathrm{Co}$ and $B u$ were about $11.6 \%, 4.2 \%, 3.8 \%$ and $9.06 \%$ respectively.

\section{Results and Discussion}

\subsection{Static tube}

A set of static tube heat transfer experiments is conducted to establish a reference data base with which to assess the influence of axial rotation on heat transfer. The detected heat transfer measurements along the radially outer edge of the static tube with the presence of twin twisted tapes for all combinations of Reynolds numbers and heat flux levels tested are found to be well correlated by $R e^{0.51}$. As shown in Fig. 3, the axial distributions of normalized static Nusselt number in terms of $N u_{0} / R e^{0.51}$, generated with all the Reynolds numbers and heat fluxes tested, converge into a tight data band at each measurement spot. The impact of buoyancy in static tube within the preset parametric range proves to be negligible. The

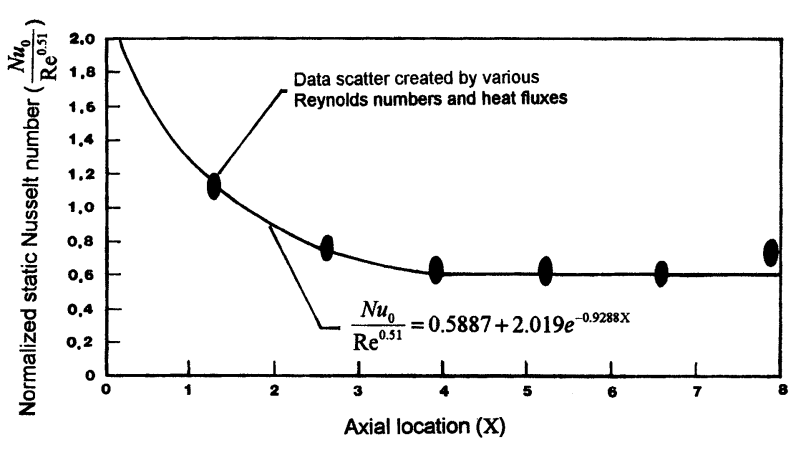

Fig. 3 Axial distributions of normalized Nusselt number along outer edge of static tube with all combinations of $R e$ and heating levels tested 
axial distributions of normalized Nusselt number in terms of $N u_{0} / R e^{0.51}$ follow the customarily forced convection behavior, in which an initially high heat transfers in the entry region due to sharp-entrance asymptotically decay to a developed value after the flow traverses about 4 tubediameters as shown in Fig. 3. The data collected in Fig. 3 is well represented by an exponential-type curve fit given by

$$
N u_{0} / R e^{0.51}=\Phi\{X\}=0.5887+2.019 e^{-0.9288 X}
$$

The variation of Prandtl number is negligible over the temperature range covered and is, in effect, absorbed into the empirical constants in Eq. (11). The data in the region of $X \geq 7.92$ is discarded in the subsequent analysis, as shown in Fig. 3, due to the usual exit loss effect present in this type of experiment. As demonstrated in Eq. (11), heat transfer augmentation in the developed flow regime due to the twin twisted tapes elevates the static Nusselt number to the level of $0.5887 R e^{0.51}$. If the thermal performance of twin twisted tape inserts in the static tube is evaluated by standardizing the Nusselt number value of tube containing twisted tapes with Dittus-Boelter value ${ }^{(17)}$ in terms of $N u_{0} / N u_{\infty}$, the averaged enhancement of $180-280 \%$ is evident in the developed flow region over the Reynolds number range 5000-15000. Note, as the $R e$ exponent in Eq. (11) is lower than the smooth-wall value of $0.8^{(17)}$, the relative enhancement in heat transfer falls when the Reynolds number increases. The reduced heat transfer augmentation as $R e$ increases has also been found in the swirl tube fitted with single twisted tape ${ }^{(14),(18)}$. Equation (11) is taken as the reference datum for subsequent comparison of the rotational effect on heat transfer.

\subsection{Rotating tube}

\subsubsection{General observations A general descrip-} tion of the data trends found in the axially rotating tube is illustrated in Fig. 4 based on one set of experiments obtained with three values of ascending buoyancy levels for a nominal Reynolds number of 5000 at Coriolis numbers of 0.1, 0.5 and 1.0. Each plot in Fig. 4 also displays the static-tube Nusselt numbers evaluated from Eq. (11) as the comparative reference to assess the rotational effect. As seen in Fig. 4, the local Nusselt number distributions along the radially outer edge of rotating tube correspond to the exponential decay pattern identified in Fig. 3. A considerable amount of heat transfer reduction from static-tube level develops in the rotating tube at Coriolis number of 0.1 . The further increase of Coriolis numbers from 0.1 to 0.5 and 1.0 incurs a subsequent heat transfer recovery, which tendency, as demonstrated in Fig. 4 (a), (b) and (c), could lead to heat transfer improvement from the statictube scenario. The importance of buoyancy interaction in the rotating tube is also shown in Fig. 4. As the heat flux is increased the streamwise gradient of flow-bulktemperature increases and this causes the buoyancy num-
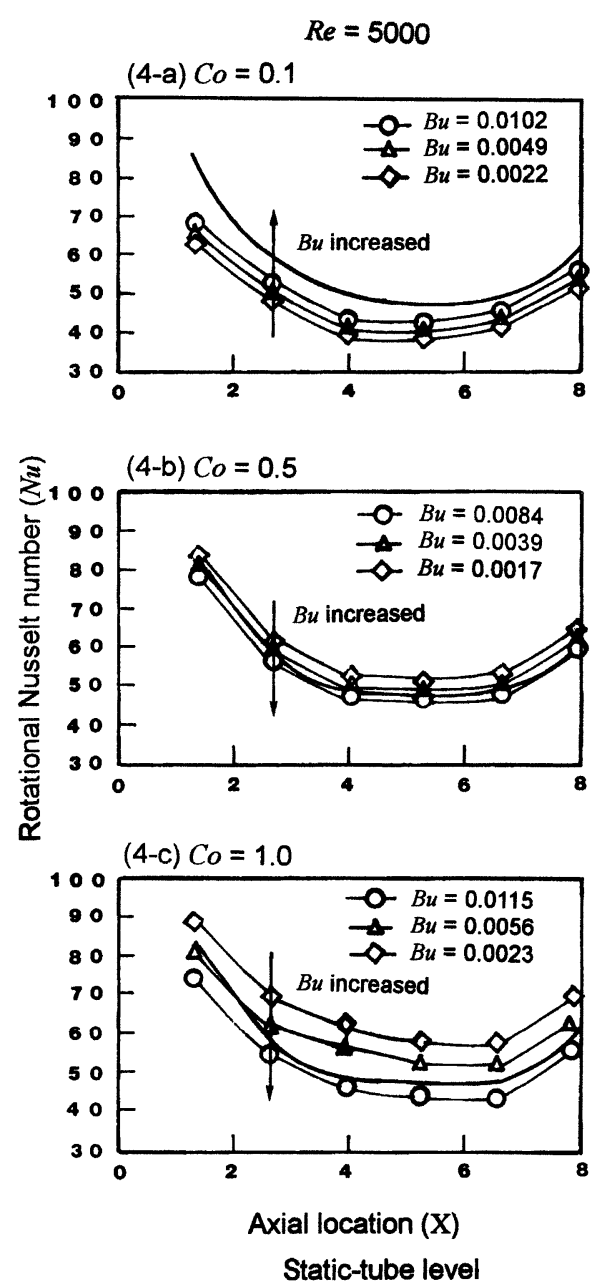

Fig. 4 Axial distributions of Nusselt number along outer edge of rotating tube with Coriolis numbers of $0.1,0.5$ and 1.0 at Reynolds number of 5000

ber to increase. The heat transfer shown in Fig. 4(a) is increased as $B u$ is increased at Coriolis number of 0.1. In this regard, Fig. 4 (b) and (c) reveal a reversing data trend that shows heat transfer reduction due to the increase of $B u$ value at Coriolis numbers of 0.5 and 1.0. A wider range of data spreads driven by varying the buoyancy number in the range about $0.002-0.01$ develops at the condition with the higher $C o$ value. The manner and degree of buoyancy impact on heat transfer therefore appear to be $C o$ dependent. This feature is as yet unexplained and will be referred to again later. However, the general pattern of local Nusselt number shown in Fig. 4 is consistent with all rotating experiments undertaken. It is also worth noting that the $\mathrm{X}$ dependence of heat transfer in the developing flow regime of rotating tube as shown in Fig. 4 (c) is different from the static scenario. The rate of streamwise decay in the rotating tube at Coriolis number of 1.0 is moderated from the static counterpart. In this regard, the flow entry condition could also be a factor that influences the interacting manner of rotational force with streamwise flow development. Thus, the rotational heat transfer results detected from the 
developed flow region are collected for deriving the heat transfer correlation in order to improve the generality of application.

3.2.2 Parametric analysis The strategic aim of deriving the physically consistent heat transfer correlation requests to identify the functional structure of Eq. (9). At zero rotational speed equation (9) must reduce to the case of static-tube solution formulated by Eq. (11). This implies that Eq. (9), applied to the present experiments, must conform to the mathematical structure

$$
\frac{N u}{N u_{0}}=\frac{\Psi\{R e, C o, B u, X\}}{\Phi\{X\} R e^{0.51}}
$$

In the radially rotating empty tube and rib-roughened duct, Morris and Chang ${ }^{(19),(20)}$ have demonstrated the isolation of Coriolis and buoyancy force-effects by treating the forced convection effect via a $R e^{n}$ term in Eq. (11), where $n$ quotes for the power index of $R e$ in the $N u_{0}$ correlation. No further presence of an explicit Reynolds number is necessary in any correlation for $N u / N u_{0}$. If this proposal is also valid for an axially rotating tube fitted with twin twisted tapes, then a significant simplification in the quest for an empirical correlation of axially rotating heat transfer ensures. A series of experiments is therefore undertaken at a fixed Coriolis number with five different Reynolds numbers. Additionally, the buoyancy numbers are carefully selected to give a reasonable constant value. As an illustrative example, these scaled rotational Nusselt numbers in terms of $N u / R e^{0.51}$ on the radially outer edge at Coriolis numbers of 0.3 and 0.5 are collected in Fig. 5.

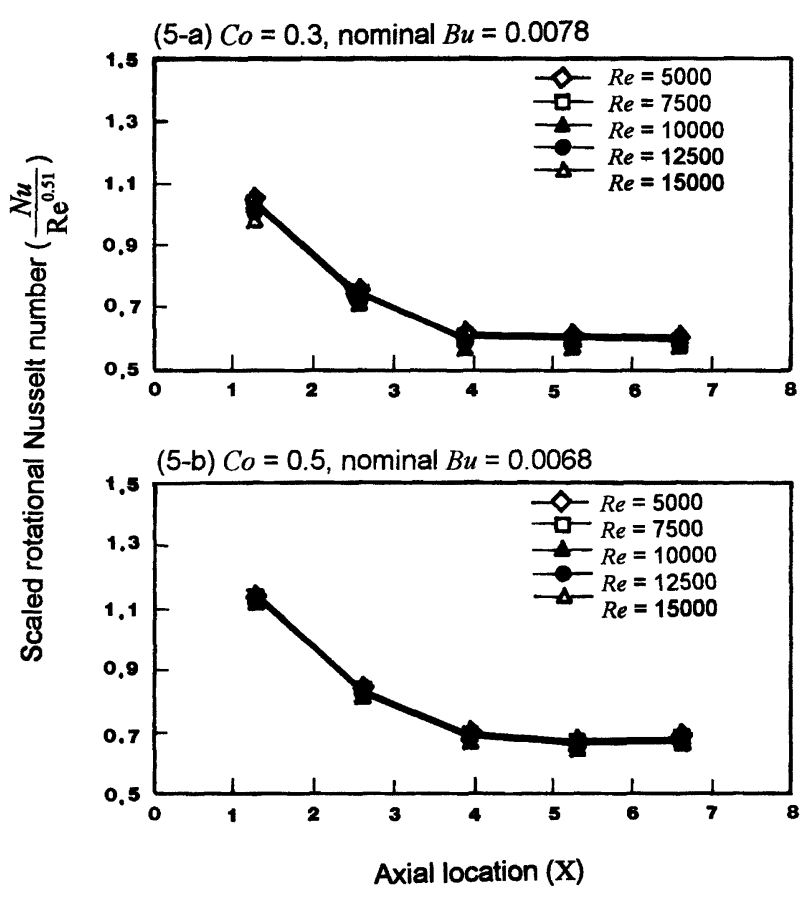

Fig. 5 Axial distributions of scaled Nusselt number along outer edge of rotating tube with a specified buoyancy level at Reynolds numbers of 5000, 7500, 10000,12500 and 15000
Each individual plot of Fig. 5 involves five Reynolds numbers with different rotational speeds in order to maintain the consistency of Coriolis number. The results demonstrate an excellent tendency to collapse the local Nusselt numbers at each measurement location onto unique curves at different Reynolds numbers. After the flow travels about 4 tube diameters as shown in Fig. 5, there appears the streamwise repeatedly Nusselt numbers which infers the developed flow region similar to the regime defined in the static tube. Thus the data generated at locations of 5.28 and $6.6 \mathrm{X}$ is collected into one data batch featuring the rotating Nusselt numbers in the developed flow regime. Subsequent parametric analysis for this developed flow regime has therefore made use of these simplified results so that Eq. (12) reduces to

$$
\frac{N u}{N u_{0}}=\phi\{C o, B u\} \text { for developed flow }
$$

Figure 4 has demonstrated the various buoyancy impacts on local heat transfer thereby confirming a strong interactive effect between the Coriolis force and buoyancy. In the regard of uncoupling buoyancy interaction to isolate the $C o$ impact on heat transfer, the finite $\beta$ value of test coolant prohibits the acquisition of the zero-buoyancy heat transfer data from experiment as the temperature difference between wall to fluid is necessary to facilitate heat transfer. However, a zero buoyancy $C o$ effect could be inferred by extrapolating a set of $\mathrm{Nu} / \mathrm{Nu}_{0}$ data from the developed flow regime taken at a specified Coriolis number with various buoyancy and Reynolds numbers. In other words, the tests conducted at different heat flux levels could be extrapolated to the zero buoyancy asymptote. This extrapolating process at Coriolis number of $0.1,0.3$, 0.5, 0.7 and 1.0 is demonstrated in Fig. 6 where the relative Nusselt number, $\mathrm{Nu} / \mathrm{Nu}_{0}$, is plotted against the combined parameter, $\mathrm{Co}^{2} \mathrm{Bu}$, for all the three axial locations in the developed flow regime. This manner of data presentation acquires the linear behavior of data-trend. Extrapolation to the zero value of $\mathrm{Co}^{2} \mathrm{Bu}$ identifies a physically realistic Nusselt number level of fully developed regime relative to $N u_{0}$ value for each Coriolis number. At the zero buoyancy asymptotic heat transfer spots revealed in Fig. 6, the relative heat transfer is reduced below the static-tube level at low values of Coriolis number but tends to recover and improve at Coriolis number in excess of about 0.3 . The positive and negative slopes of each data trends typified in the individual plot of Fig. 6, respectively, reflect the improving and impeding buoyancy effect on heat transfer with the degree of impact featured by the magnitude of the slope. A systematic variation of the slopes of these data trends collected in Fig. 6 is demonstrated, which shows a gradually reduction from positive values at Coriolis numbers of 0.1 and 0.3 to the negative values for the cases of $C o=0.5,0.7$ and 1.0. Therefore, the increase of Coriolis number from 0.1 to 1.0 could yield the buoyancy impact from improv- 


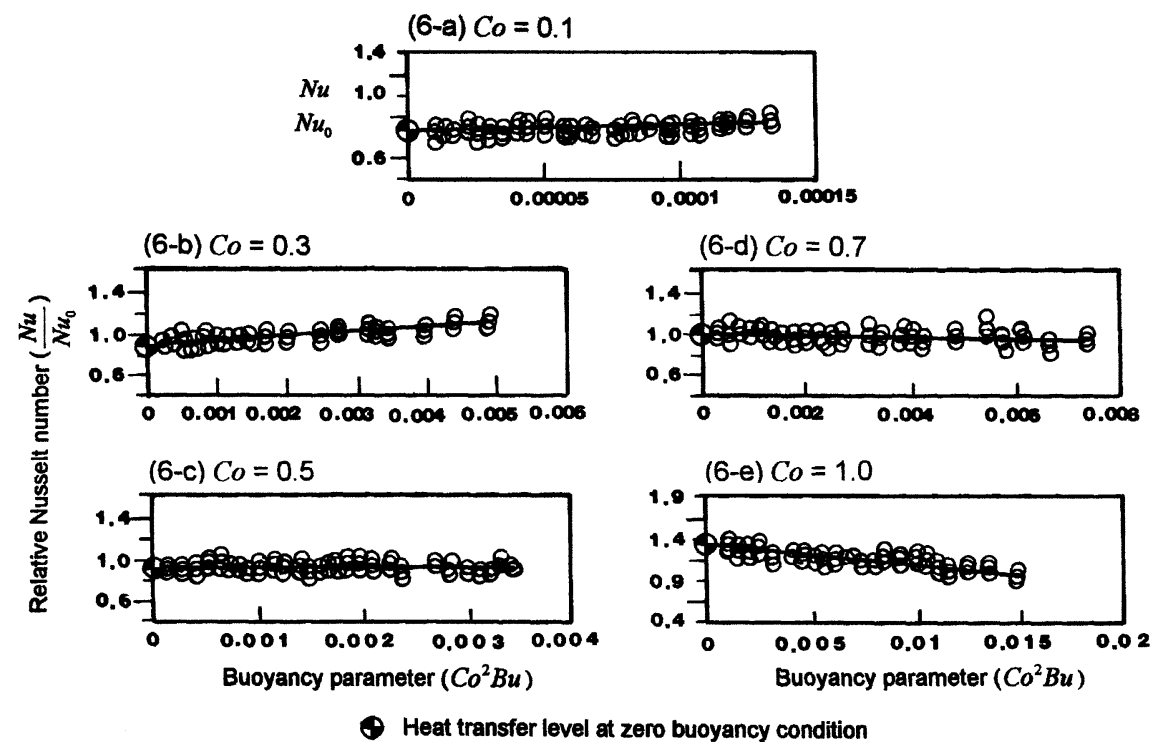

Fig. 6 Variations of relative Nusselt number with buoyancy parameter at fixed Coriolis numbers

ing to impeding heat transfer in an axially rotating tube fitted with twin twisted tapes. The convergence of data points and the revealed data trend shown in Fig. 6 have led to the proposal of a possible correlation form for $\mathrm{Nu} / \mathrm{Nu}$ as

$$
\frac{N u}{N u_{0}}=f_{C}\{C o\}+f_{B}\{C o\} \times C o^{2} B u
$$

where $f_{C}$ and $f_{B}$ respectively quantify the individual $C o$ impact and the inter-correlated $C o-B u$ effect on heat transfer. As the flow in a rotating tube fitted with twisted plate could have a very complex three-dimensional structure which could lead to circumferential heat transfer variations, the $f_{C}$ and $f_{B}$ values could be accordingly varied with the circumferential location. As the present study detects the axial heat transfer variations along the outer edge of the rotating tube, the correlation derived is applicable to evaluate the heat transfer along the outer edge of present rotating tube. The functional values of $f_{C}$ and $f_{B}$ are respectively collected from the asymptotic zero-buoyancy heat transfer levels and the slopes of data trends unraveled in Fig. 6. The involvement of zero rotational speed solution requires $f_{C}$ value to be unity and the self-vanished $f_{B} \times \mathrm{Co}^{2} \mathrm{Bu}$ value when $\mathrm{Co}$ becomes zero. Figure 7 shows the manner in which $f_{C}$ and $f_{B}$ varied with the Coriolis number for the developed flow regime. The data trend unraveled in Fig. 7 (a) could be treated as the individual Coriolis force effect on heat transfer at the limiting condition of vanished wall-to-fluid temperature difference. Unlike most of the results found in an axially rotating empty tube where the influences of Coriolis force on heat transfer remains as the secondary effect that is only considerable in the developing flow region ${ }^{(1)-(7)}$, the swirling flow structures created by the twin twisted tapes in the rotating tube has promoted the Coriolis force effect into a dominant
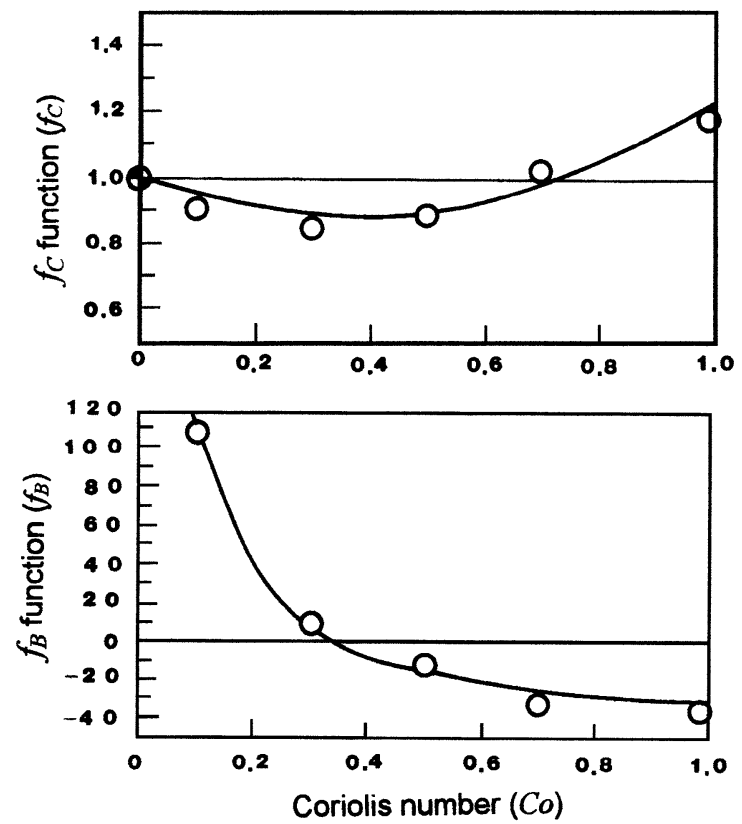

Fig. 7 Variations of $f_{C}$ and $f_{B}$ functional values with Coriolis number

feature. The initial heat transfer reduction from the statictube scenario, followed by the subsequent heat transfer recovery is originated from the Coriolis-force impacts. The reversal of buoyancy effect from improving to impeding heat transfer could occur when $C o>0.4$ as identified in Fig. 7 (b). Led by the observation of data trends depicted in Fig. 7 and the physical constraints required for the involvement of static-tube solution, the $f_{C}$ and $f_{B}$ values are correlated by the following equations

$$
\begin{aligned}
& f_{C}=1-0.6612 \times C o+0.8858 \times C o^{2} \\
& f_{B}=-31.53+285 e^{-6.956 C o}
\end{aligned}
$$




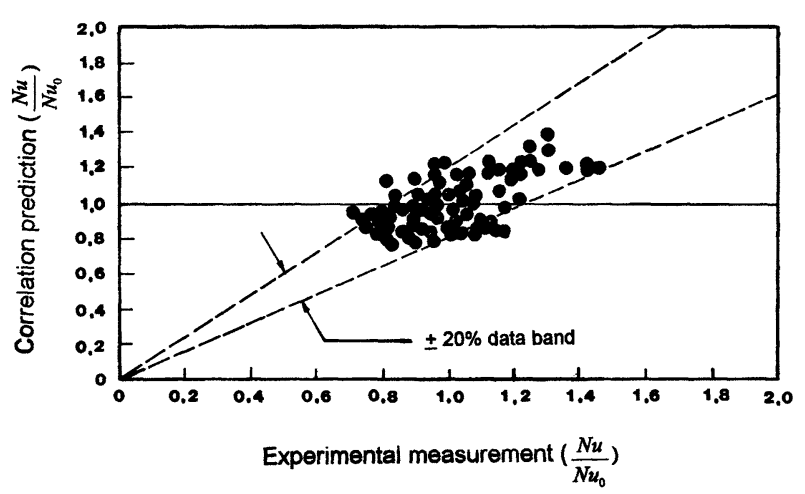

Fig. 8 Comparison of experimental measurements with correlation predictions in terms of relative Nusselt number

As the static-tube Nusselt number, $N u_{0}$, for the developed flow regime is evaluated by $0.5887 R e^{0.51}$ in Eq. (11), the heat transfer correlation in the developed flow regime of an axially rotating tube fitted with the present tested twin twisted tapes is accordingly acquired as

$$
\begin{aligned}
N u & =0.5887 \times R e^{0.51} \times\left\{1-0.8401 \times C o+1.218 \times C o^{2}\right. \\
& \left.+\left(-31.53+285 e^{-6.956 C o}\right) \times C o^{2} \times B u\right\}
\end{aligned}
$$

The prediction of heat transfer in an axially rotating tube fitted with twin twisted tapes is a formidable task owing to the complex interaction between the twisted plate induced swirls and the combined influence of Coriolis and buoyancy forces. The present study has devised a methodology to uncouple these effects via a systematic experimental strategy along with a detailed procedure of data analysis. The result is the empirical correlation embodied in Eq. (17) which could be used to estimate heat transfer in the developed flow regime at the outer edge of an axially rotating tube fitted with twin twisted tapes. Comparing all the experimental measurements with the correlative predictions based on Eq. (17) has performed as a review for the overall success of this empirical proposal. Over the entire range of parametric conditions studied, $90 \%$ of the present experimental data in terms of $N u / N u_{0}$ is found to agree within $\pm 20 \%$ of the correlation proposed. Figure 8 summarizes the comparison of experimental measurements and correlative results for all three axial locations in the developed flow regime. As seen, most of data falls in the region of $0.8-1.0$. The heat transfer reduction to the level less than $N u_{0}$ owing to the rotational effect requires the specific attention when this enhanced cooling measure is applied for cooling of rotor windings. Consider the complexities of the flow and heat transfer involved, the proposed correlation could offer a good indication of the individual and combined effects of $\mathrm{Re}, \mathrm{Co}$ and $\mathrm{Bu}$ on heat transfer in an axially rotating tube fitted with twin twisted tape inserts.

\section{Conclusions}

An experimental study of heat transfer in an axially rotating tube fitted with twin twisted tapes was performed to unravel the effect of rotation on the heat transfer performance. With considerations of physical and theoretical consistencies, this paper illustrates an experimental strategy, from the design of apparatus to the detailed analysis of the resulting derived data, which could lead to the heat transfer correlation and uncoupling each dynamic force effects involved. The net result has been an empirical equation embodied in Eq. (17), which may be used to estimate rotational Nusselt numbers in the developed flow regime of an axially rotating tube fitted with the present twin twisted tapes. The detailed measurements of local heat transfer along the radially outer edge have been acquired and analyzed. The swirling flow structures generated by the twin twisted tapes incur considerable Coriolis force effects throughout the entire rotating tube, which effect interacts with buoyancy to provide significant heat transfer modification from the static-tube scenario. In this respect the individual effects of Coriolis and buoyancy have been independently assessed and the zero buoyancy condition evaluated by extrapolation. With test system been pressurized, the range of the non-dimensional parameters governing this heat transfer regime have been considerably extended. In what follows, the important points those have been identified from this experimental investigation are summarized.

1. The spatial distributions of normalized Nusselt number of $N u_{0} / R e^{0.51}$ in static tube gradually decrease from flow entrance towards the asymptotic value in the developed flow regime, which is found to be 1.8 to 2.8 times of the empty-tube Dittus-Boelter level in the Re range of $5000-15000$. The relative heat transfer enhancement in terms of $N u / N u_{\infty}$ falls when the Reynolds number increases. The static-tube heat transfer data is correlated with $R e^{0.51}$ in the form of Eq. (11).

2. The isolation of forced convection from Coriolis and buoyancy effects in the rotating tube using the $R e^{0.51}$ scaling idea at each measurement location has been successfully demonstrated. This enables a great simplification of the quest for a correlating equation along the lines of Eq. (12) and allows identifying the individual $C o$ and $B u$ impacts on heat transfer. By extrapolating the experimental data to the implied zero buoyancy scenario, the isolated Coriolis force effect initially reduces heat transfer from the static-tube level which is followed by a subsequent recovery that could lead to heat transfer improvement when $C o$ increases from 0.1 to 1.0 .

3. The buoyancy impact on heat transfer in the present rotating tube is $C o$ dependent, which could be reversed from improving to impeding heat transfer when $C o$ value is above 0.4 .

4. The proposed empirical correlation for the developed flow regime in the axially rotating tube fitted with twin twisted tapes is physically consistent and permits the 
evaluations of individual and interactive effects of forcedconvective inertia, Coriolis force and centripetal buoyancy on heat transfer.

\section{References}

( 1 ) Morris, W.D., Heat Transfer and Fluid Flow in Rotating Coolant Channels, (1981), Research Studies Press, Wiley, New York, ISBN 0471101214.

( 2 ) Morris, W.D., Laminar Convection in a Heated Vertical Tube Rotating about a Parallel Axis, J. Fluid Mech., Vol.21, Part 3 (1965), p.453.

( 3 ) Woods, J.L. and Morris, W.D., An Investigation of Laminar Flow in the Rotor Windings of DirectlyCooled Electrical Machines, J. Mech. Eng. Sci., Vol.16 (1974), pp.408-417.

( 4 ) Woods, J.L., Heat Transfer and Flow Resistance in a Rotating Duct System, Ph.D. Thesis, University of Sussex, Falmer, England, (1975).

( 5 ) Majumdar, A.K., Morris, W.D., Skiadaressis, D. and Spalding, D.B., Heat Transfer in Rotating Ducts, Mech. Engng. Bull., Vol.8 (1977), pp.87-95.

( 6 ) Dias, F.M., Heat Transfer and Resistance to Flow in Rotating Square Tubes, Ph.D. Thesis, University of Sussex, Falmer, England, (1978).

( 7 ) Woods, J.L. and Morris, W.D., A Study of Heat Transfer in a Rotating Cylindrical Tube, ASME J. Heat Transfer, Vol.102 (1980), pp.612-616.

( 8 ) Mori, Y. and Nakayama, W., Convective Heat Transfer in a Rotating Radial Circular Pipe, (2nd Report, Turbulent Region), Int. J. Heat Mass Transfer, Vol.14 (1971), pp.1807-1824.

( 9 ) Mahadevappa, M., Rao, V.R. and Sastri, V.M.K., Numerical Study of Steady Laminar Fully Developed Fluid Flow and Heat Transfer in Rectangular and Elliptical Ducts Rotating about a Parallel Axis, Int. J. Heat Mass Transfer, Vol.39 (1996), pp.867-875.

(10) Date, A.W., Prediction of Fully-Developed Flow in a Tube Containing a Twisted-Tape, Int. J. Heat Mass
Transfer, Vol.17 (1974), pp.845-859.

(11) Hong, S.W. and Bergles, A.E., Augmentation of Laminar Flow Heat Transfer in Tubes by Means of TwistedTape Inserts, ASME J. Heat Transfer, Vol.98 (1976), pp.251-256.

(12) Manglik, R.M. and Bergles, A.E., Heat Transfer and Pressure Drop Correlations for Twisted-Tape Inserts in Isothermal Tubes: Part I — Laminar Flows, ASME J. Heat Transfer, Vol.115 (1993), pp.881-889.

(13) Manglik, R.M. and Bergles, A.E., Heat Transfer and Pressure Drop Correlations for Twisted-Tape Inserts in Isothermal Tubes: Part II — Transition and Turbulent Flows, ASME J. Heat Transfer, Vol.115 (1993), pp.890-896.

(14) Agarwal, S.K. and Raja, R.M., Heat Transfer Augmentation for the Flow of a Viscous Liquid in Circular Tubes Using Twisted Tape Inserts, Int. J. Heat Mass Transfer, Vol.39 (1996), pp.3547-3557.

(15) Ray, S. and Date, A.W., Laminar Flow and Heat Transfer through Square Duct with Twisted Tape Insert, Int. J. Heat Fluid Flow, Vol.22 (2001), pp.460-472.

(16) Editorial Board of ASME J. Heat Transfer, Journal of Heat Transfer Policy on Reporting Uncertainties in Experimental Measurements and Results, ASME J. Heat Transfer, Vol.115 (1993), pp.5-6.

(17) Dittus, F.W. and Boelter, L.M.K., University of California Publications on Engineering, University of California, Berkeley, CA, Publ. Eng., Vol.2 (1930), p.443.

(18) Chang, S.W., Heat Transfer of Parallel-Mode Reciprocating Tube Flow with Twisted-Tape Insert for Piston Cooling Application, ASME Journal of Gas Turbine and Power, Vol.123 (2002), pp.146-156.

(19) Morris, W.D. and Chang, S.W., Heat Transfer in a Radially Rotating Smooth-Walled Tube, The Aeronautical Journal, Vol.102 (1998), pp.277-285.

(20) Chang, S.W. and Morris, W.D., Heat Transfer in a Radially Rotating Rib-Roughened Square Duct, Int. J. Thermal Sciences, Vol.42 (2003), pp.267-282. 